\title{
Effect of localized helium ion irradiation on the performance of synthetic monolayer $\mathrm{MoS}_{2}$ field-effect transistors
}

\author{
Jakub Jadwiszczak ${ }^{1}$, Pierce Maguire ${ }^{1}$, Conor P. Cullen², Georg S. Duesberg ${ }^{2,3}$ \\ and Hongzhou Zhang ${ }^{*}$
}

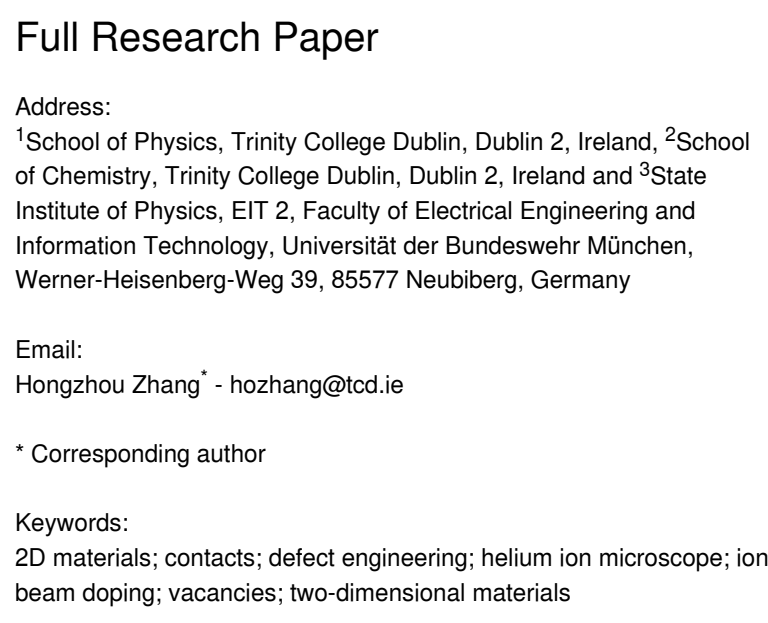

Beilstein J. Nanotechnol. 2020, 11, 1329-1335.

https://doi.org/10.3762/bjnano.11.117

Received: 06 May 2020

Accepted: 19 August 2020

Published: 04 September 2020

This article is part of the thematic issue "Ten years of the helium ion microscope".

Guest Editors: G. Hlawacek and A. Wolff

(C) 2020 Jadwiszczak et al.; licensee Beilstein-Institut.

License and terms: see end of document.

\begin{abstract}
Helium ion irradiation is a known method of tuning the electrical conductivity and charge carrier mobility of novel two-dimensional semiconductors. Here, we report a systematic study of the electrical performance of chemically synthesized monolayer molybdenum disulfide $\left(\mathrm{MoS}_{2}\right)$ field-effect transistors irradiated with a focused helium ion beam as a function of increasing areal irradiation coverage. We determine an optimal coverage range of approx. $10 \%$, which allows for the improvement of both the carrier mobility in the transistor channel and the electrical conductance of the $\mathrm{MoS}_{2}$, due to doping with ion beam-created sulfur vacancies. Larger areal irradiations introduce a higher concentration of scattering centers, hampering the electrical performance of the device. In addition, we find that irradiating the electrode-channel interface has a deleterious impact on charge transport when contrasted with irradiations confined only to the transistor channel.
\end{abstract}

\section{Introduction}

Layered two-dimensional (2D) semiconductors have come to the fore in recent years as promising candidates for the implementation of flexible, transparent, and low-power electronics. In particular, transition metal dichalcogenides (TMDs), such as molybdenum disulfide $\left(\mathrm{MoS}_{2}\right)$, have demonstrated impressive on/off ratios (approx. 107 ) in field-effect transistors (FETs), while maintaining carrier mobilities that may be adequate for commercial applications [1,2]. At the same time, advances in chemical vapor deposition (CVD) techniques have allowed for the reliable millimeter-scale synthesis of well-performing monolayer TMD films [3-5], leading to viable large-scale integration of on-chip TMD FETs. With device miniaturization, it 
becomes key to understand the impact of defects such as chalcogen vacancies on the electrical transport properties of FETs based on 2D semiconductors. This is particularly crucial for device applications in radiation-rich environments (e.g., space satellite technologies), since defects can be introduced by ionizing particle irradiation while the devices are in continuous operation.

Recently, noble gas ion beam irradiation has opened the field to the exploration of nanometer-scale structural modifications of TMD devices [6-8]. The localized formation of defects by focused ion beam irradiation has been shown to induce unusual electronic properties in monolayer TMDs, such as pseudometallic phase transitions in $\mathrm{MoS}_{2}$ and $\mathrm{WSe}_{2}[9,10]$, resistive switching in $\mathrm{MoS}_{2}$ [11], as well as enhanced out-of-plane charge transfer in $2 \mathrm{D}$ graphene/ $\mathrm{WSe}_{2}$ heterostructures [12] Energetic light ions are known to preferentially sputter chalcogen atoms from TMDs while retaining an adequate micrometer-scale structural integrity for irradiation doses up to approx. $10^{16}$ ions $\mathrm{cm}^{-2}$ [13-17], as well as good electrical conductivity for up to approx. $10^{18}$ ions $\mathrm{cm}^{-2}[9,10,18]$. Sulfur vacancies (SVs) and the formation of a dislocation-divacancy complex can lead to significant n-doping in $\mathrm{MoS}_{2}$ [19], which shifts the threshold voltage $\left(V_{\text {th }}\right)$ of the FET to higher negative gate biases $[20,21]$. These complex states may also improve the carrier mobility across a given gate bias range by forming stable impurity bands near the conduction band [22]. Some theoretical studies suggest, however, that individual SVs ought to act as electron acceptors in $\mathrm{MoS}_{2}[23,24]$. As the spread of a typical focused $\mathrm{He}^{+}$ion probe is several nanometers, the formation of other defects in the irradiated 2D crystal lattice is also expected $[25,26]$, which may bring about the often-observed negative shifts of the $\mathrm{MoS}_{2}$ FET threshold voltage after ion irradiation. Such n-type doping behavior achieved by selective ion sputtering in thin film transistors has also been observed in $\mathrm{He}^{+}-$ irradiated InGaZnO devices [27]. This irradiation-induced carrier activation depends not only on the fluence of the ion beam, but also on the absolute number of defects that can be introduced. Therefore, the creation of defects in TMD FETs may serve to improve charge transport and tune the device performance $[10,20,28]$, while the effective donor concentration and the introduced scattering potentials need to be tailored accordingly $[29,30]$. Thus far, these studies have focused either on spatially confined defect generation, or full-channel modifications in TMD FETs. A research space exists for exploring the intermediate regime between these two extrema by finely controlling the area over which defects are seeded. For practical devices, it is also important to consider the effect of particle irradiation on the deposited metal-semiconductor contact interface. Recent work has shown that irradiation-induced heating of the electrode area can reverse majority carrier polarity in $\mathrm{MoTe}_{2}$ [31], while pre-treatment with a broad-beam argon ion source can decrease the contact resistance of $\mathrm{Ni}-\mathrm{MoS}_{2}$ two-fold [32]. In this paper, we investigate the effect of the defect population on the performance of $\mathrm{MoS}_{2}$ FETs via varying the area of ion irradiation in the FET channel. We also examine the performance of devices upon irradiation of one of the electrical contact interfaces.

\section{Experimental}

Monolayer $\mathrm{MoS}_{2}$ samples were synthesized using a CVD microreactor method, described in detail in [33], directly on $285 \mathrm{~nm} \mathrm{SiO}_{2} / \mathrm{Si}$ substrates, which also served as the back-gate in the FET configuration. $\mathrm{MoS}_{2}$ flakes were contacted with electrodes using standard electron beam lithography on polymethyl methacrylate (PMMA) resist, followed by lift-off in acetone and metal deposition by evaporation $(5 \mathrm{~nm}$ Ti and $35 \mathrm{~nm}$ $\mathrm{Au}$ ). Electrical testing was carried out using a dual-channel source-measure unit connected to tungsten micromanipulator probes (Imina miBot) in the vacuum chamber of a customized Zeiss EVO scanning electron microscope (SEM). Prior to testing, the devices were outgassed at a pressure of approx. $10^{-5}$ mbar for $12 \mathrm{~h}$ to minimize surface adsorbates. Helium ion irradiations were carried out at a beam energy of $30 \mathrm{keV}$ and $\mathrm{He}$ gas pressure of $2 \times 10^{-6}$ Torr, using a Zeiss Nanofab microscope. The fabricated $\mathrm{MoS}_{2}$ FETs were placed in the helium ion microscope chamber (after initial electrical testing to confirm functionality) and were irradiated with the stage tilt angle set to $0^{\circ}$. At this angle of incidence, the helium ion beam ought to produce sulfur vacancies chiefly in the bottom sulfuric layer of the $\mathrm{SiO}_{2}$-supported $\mathrm{MoS}_{2}$ flake [34]. The average recorded beam current throughout the irradiations was $37.5 \pm 0.4 \mathrm{pA}$, and the probe size was determined at approx. $7 \mathrm{~nm}$ [9]. The areal ion dose delivered to each sample was maintained at approx. $10^{17}$ ions $\mathrm{cm}^{-2}$, with a step size of $1 \mathrm{~nm}$ and a dwell time of $4.3 \mu$ s throughout the duration of a unidirectional probe scan. Post-irradiation SEM imaging was carried out in a Zeiss Supra microscope using the in-lens detector at a low beam energy of less than $5 \mathrm{keV}$ to ensure a strong surface sensitivity. Figure 1a shows a sketch of the experimental geometry. A SEM micrograph of a typical irradiated device is also presented in Figure 1b. The marked distances $W$ and $L$ denote the width of the irradiated region and the length of the FET channel, respectively. $L$ was kept constant at $5 \mu \mathrm{m}$ in this work, while $W$ was varied to obtain a designated irradiation-to-channel ratio, $I_{\mathrm{R}}=W / L$. Following the irradiation and electrical testing, these dimensions were re-measured in the SEM to obtain accurate $I_{R}$ values in case of beam drift throughout the procedure.

\section{Results and Discussion}

As shown in Figure 1c, the localized irradiation (dose $=$ $10^{17}$ ions $\mathrm{cm}^{-2}, I_{\mathrm{R}}=7 \%$ ) causes a notably higher electrical 
(a)

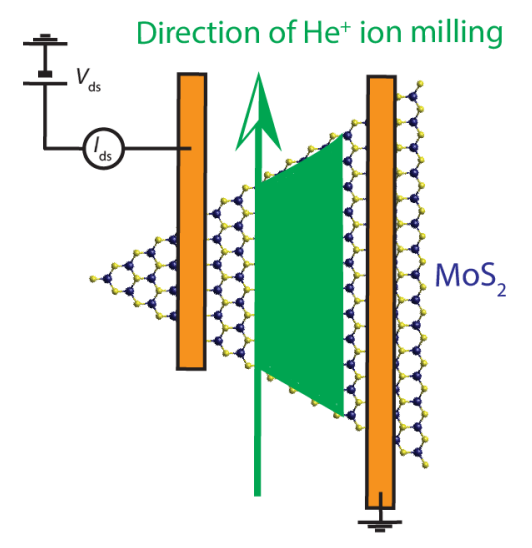

(c)

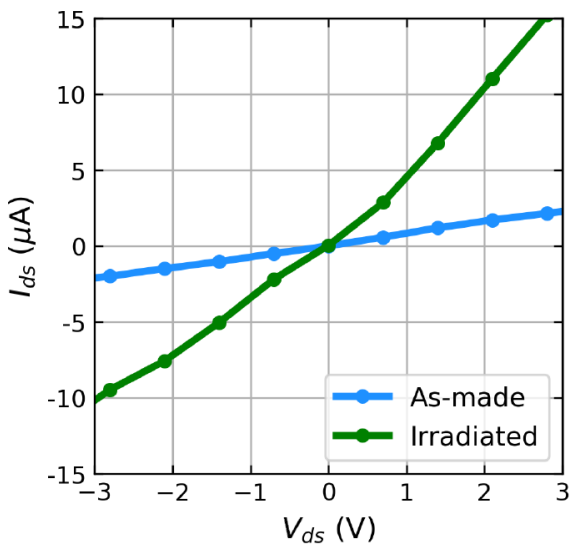

(b)

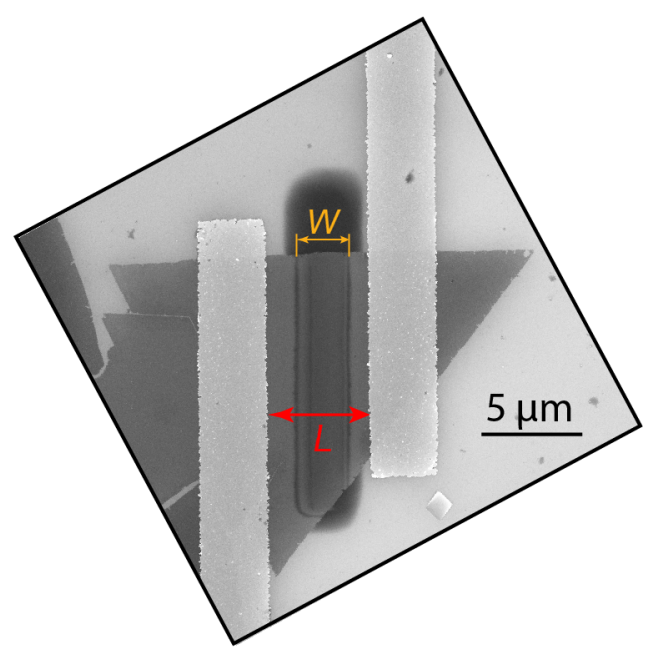

(d)

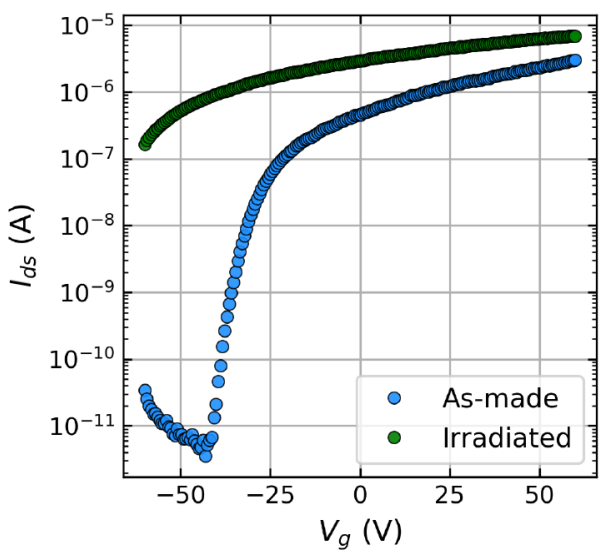

Figure 1: Experimental design and basic electrical characteristics. (a) Sketch demonstrating the irradiation strategy on contacted CVD-grown monolayer $\mathrm{MoS}_{2}$ devices. The green area marks the designed irradiation area. (b) SEM image of an irradiated device. $W$ marks the width of the exposed region, while $L$ is the length of the FET channel. $L$ is $5 \mu \mathrm{m}$ in the image. (c) $I-V$ curve of a device after irradiation (corresponding to $I_{R}=7 \%$ ).

(d) Transfer curve of the same device demonstrating reduced gate tunability and a higher electrical conductance after $\mathrm{He}^{+}$ion irradiation.

conduction to emerge in the monolayer $\mathrm{MoS}_{2}$ device. The output current $\left(I_{\mathrm{ds}}\right)$ increases approx. fivefold for the same drain-source bias $\left(V_{\mathrm{ds}}\right)$ when compared with the non-irradiated device. After the helium ion beam exposure, the transfer characteristics, Figure 1d, reveal a clearly reduced electrostatic response to the applied gate field. The FET channel cannot be effectively turned off in the tested bias range, with significant drain currents persisting even at $V_{\mathrm{g}}=-60 \mathrm{~V}$. This leads to a sharp increase in the subthreshold swing and a large shift of $V_{\text {th }}$ towards negative gate bias values $(\Delta>10 \mathrm{~V})$, thus deepening the depletion-mode n-type channel functionality. This observed near-degenerate doping behavior is typical of an increased concentration of sulfur vacancies in defect-rich $\mathrm{MoS}_{2}[9,20,21]$. We note here, that a recent high-energy irradiation study has also called for attention to charge traps generated in the underlying oxide, as the source of donor states in the FET channel [35]. Further studies on flakes decoupled from the sub- strate need to be performed to clarify the exact origin of the threshold voltage shift in TMDs irradiated at moderate beam energies.

The effects of increasing $I_{\mathrm{R}}$ are evident from changes to the recorded output and transfer characteristics in Figure 2a,b. The data are organized into three groups, marked blue, green, and red, corresponding to small (2-18\%), medium (28-41\%) and large $(48-76 \%) I_{\mathrm{R}}$ values, respectively. The $I_{\mathrm{ds}}-V_{\mathrm{ds}}$ curves, taken at $V_{\mathrm{g}}=0 \mathrm{~V}$, demonstrate an increase in the channel conductance between the $I_{\mathrm{R}}=2 \%$ and $I_{\mathrm{R}}=7 \%$ irradiations, followed by a continuing drop when $I_{\mathrm{R}}>18 \%$. For small values of $I_{\mathrm{R}}$ (blue curves in Figure 2b), the FET experiences a large $V_{\text {th }}$ shift $(\Delta>10 \mathrm{~V})$ to negative gate biases, a lowered on/off ratio (approx. $10^{2}$ ), and an increased conductance relative to the untreated device. As $I_{\mathrm{R}}$ is increased into the green (28-41\%) and red $(48-76 \%)$ groups, the device conductance drops 
(a)

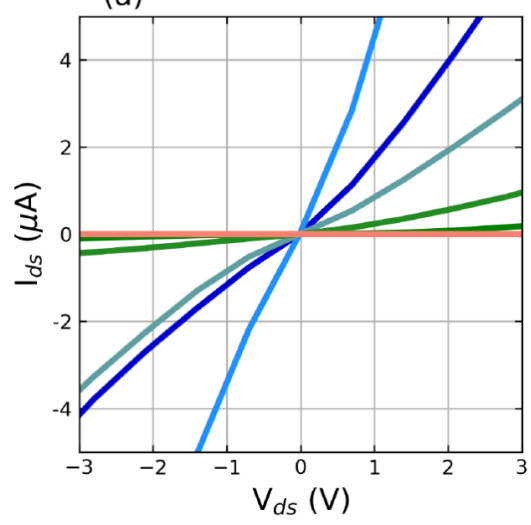

(c)

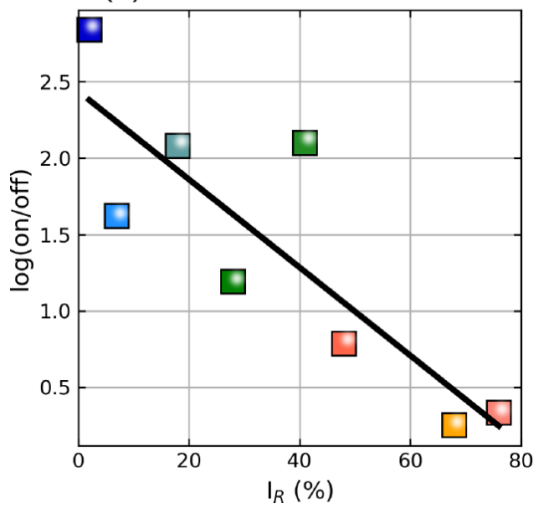

(b)

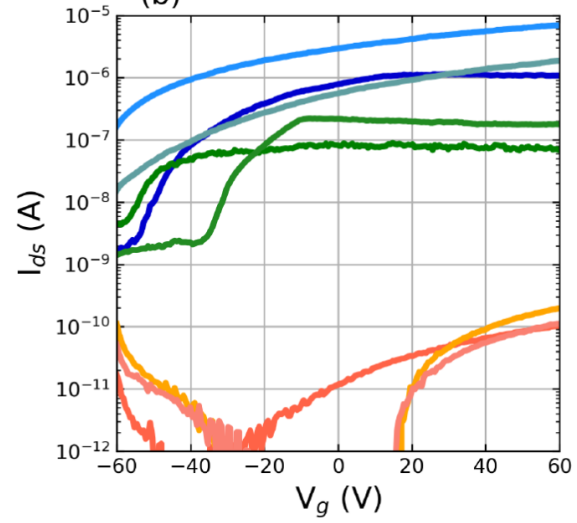

(d)

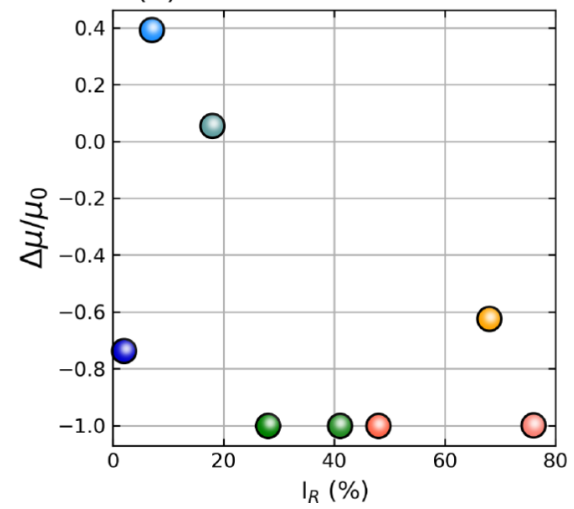

$I_{\mathrm{R}}=W / L$

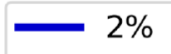

$7 \%$

$18 \%$

$28 \%$

$41 \%$

$48 \%$

$60 \%$

$76 \%$

Figure 2: Effects of changing the irradiated area on the performance of monolayer $\mathrm{MoS}_{2}$ FETs. Note that all the plots share the same color legend on the right. (a) $I-V$ and (b) gate sweeps of different devices with varying $I_{R}$. (c) Semi-log plot of the extracted electron branch on/off ratios corresponding to each gate curve in (b). The black line is a linear fit to the semi-log data. (d) Changes in the field-effect mobility, $\mu$, relative to as-made device mobilities, $\mu_{0}$, extracted from transfer curves in (b).

heavily, accompanied by positive-bias $V_{\text {th }}$ shifts, while the on/off ratio is also seen to decrease roughly exponentially. This is demonstrated in Figure 2c, which tracks the log-transformed on/off ratio as a function of $I_{\mathrm{R}}$ with a good linear correlation $(R=-0.85)$ from the semi-logarithmic fit. This suggests that an optimal ion irradiation strategy for improving the channel conductance, i.e., when the areal channel exposure is close to $10 \%$, needs to be a balance between introducing significant n-dopant densities and suppressing the unwanted crystal structure amorphization which results from high irradiation doses. At high $I_{\mathrm{R}}$ values (red group), we observe the emergence of a weak ambipolar response in our transfer curves. At these ratios, the device starts to enter a regime where more than half of the channel has been treated with the ion beam. Thus, we expect a dominant contribution of oxygen-containing atmospheric adsorbates (known p-type dopants in $\mathrm{MoS}_{2}$ ) in saturating the vacancy sites created by the ion beam, allowing for residual hole conduction in the newly formed effective medium channel [3638]. However, the role of other impurities such as hydrogen and carbon from adventitious surface hydrocarbons in the observed p-doping ought to also be considered in future studies. For a given delivered dose, the ion beam provides a high concentration of effective adsorption sites for atmospheric p-dopants (or adventitious hydrocarbons) within the area defined by the spread of the Gaussian probe extension. The probe has spatially trailing lower-dose tails, the damage of which extends to more than $10 \mathrm{~nm}$ and may induce additional n-doping with no complementary adsorbant saturation, i.e., by creating unsaturated SVs in the bottom sulfuric layer [34,39]. A larger irradiation area will thus provide more effective p-doping sites while stifling the n-doping response as the probe tails begin to extend past the FET channel. In addition, low-dose trail areas will be irradiated repeatedly by adjacent line scans, increasing the effective delivered dose and shifting that region to a more acceptor-rich area. This leads to the observed ambipolar gate response for large $I_{\mathrm{R}}$ values. The formation of these back-toback heterojunctions may result in unconventional charge transport phenomena if the dose of the irradiation is varied across several orders of magnitude [9], which we leave for future studies. 
Figure $2 \mathrm{~d}$ charts the effect of $I_{\mathrm{R}}$ on the change in the carrier field-effect mobility, $\mu$, of the irradiated device relative to its as-fabricated mobility, $\mu_{0}$. Extracted from the linear region of the transfer curve, $\mu$ is seen to improve by up to $40 \%$ in two out of three devices in the blue region. For the green and red regions of $I_{R}, \mu$ is always seen to worsen as the area of the irradiated channel is increased. $\mu$ is expected to drop heavily as the rate of electron scattering rises with the increased defective channel area. The improved mobility at $I_{\mathrm{R}} \approx 10 \%$ indicates the screening of scattering centers by the increased carrier concentrations induced by SV doping, which leads to the increased conductance observed in Figure 2a. We note that our measurements were performed in a two-probe geometry. Thus, the absolute values of $\mu$ extracted here (approx. $1 \mathrm{~cm}^{2} \mathrm{~V}^{-1} \mathrm{~s}^{-1}$ ) are limited by the contact resistance between the gold and the $\mathrm{MoS}_{2}$ [40].

We now consider the effect of irradiating the metal-semiconductor interface. We treated two FETs within each $I_{\mathrm{R}}$ regime. For one of the devices in each pair a single electrode interface was also irradiated. SEM images in Figure 3a show the irradiated channel areas colored in green and the non-irradiated $\mathrm{MoS}_{2}$ channel areas in red, on example devices in the high $I_{\mathrm{R}}$ regime. In all three $I_{\mathrm{R}}$ regimes, allowing one of the electrode- $-\mathrm{MoS}_{2}$ interfaces to be damaged by the helium ion beam leads to a drop in the device conductance, as evident in Figure $3 b$ for the repre- sentative case of medium $I_{\mathrm{R}}$ values. Moreover, the conductance of the device where the electrode- $\mathrm{MoS}_{2}$ interface was treated with the ion beam is lower than the conductance of the as-fabricated non-irradiated device. The transfer curves in Figure $3 \mathrm{c}$ indicate that as the device approaches the strong inversion regime, the electrode-channel interface damage (green curve) inhibits high drain currents in the FET, in contrast to the case of no electrode damage (purple curve).

We simulated the atomic vacancy yield per each delivered ion as a function of target penetration depth on the $35 \mathrm{~nm}-\mathrm{Au} /$ $5 \mathrm{~nm}-\mathrm{Ti} / 0.7 \mathrm{~nm}-\mathrm{MoS}_{2} / 285 \mathrm{~nm}-\mathrm{SiO}_{2}$ stack [41]. As evident from Figure 3d, the sulfur sputtering yield at the Ti-MoS 2 interface is very close to that of unencapsulated $\mathrm{MoS}_{2}$ [15], indicating notable damage to the interface at this delivered dose. It may be expected that an increase in the Schottky barrier height will occur if the normally pinned Fermi level [42] is now a function of the physical state of the beam-altered metal-semiconductor interface. Ion beam pre-treatment of the contact region before metal deposition increases the concentration of dangling bonds available for molecular hybridization when the contact metal is deposited [32]. As we are treating an already hybridized interface, we suspect that the formation of point defects therein, such as migrated interstitials and antisite defects, will serve to trap carriers at the interface and will reduce the crowded injection current at the contact [43]. This (2)

(d)

(a)
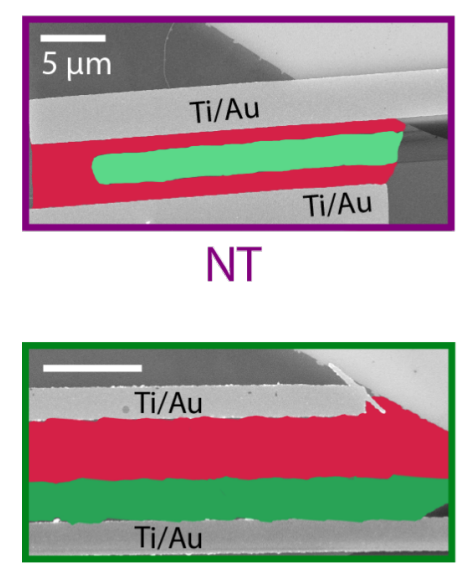

T
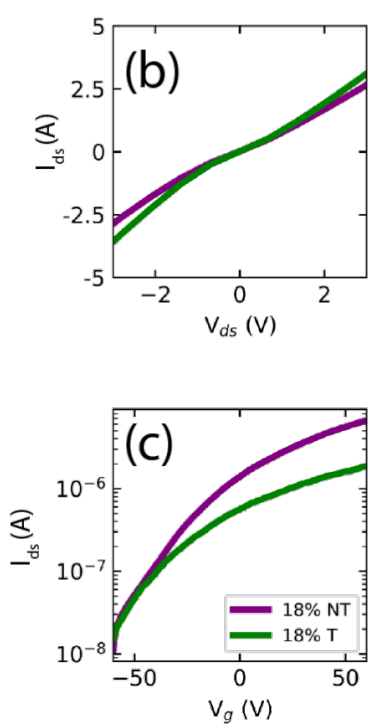

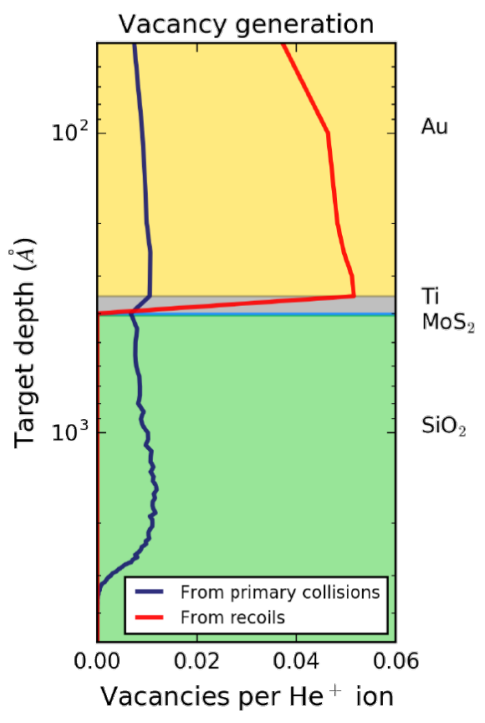

Figure 3: (a) Example SEM images of electrode non-touching (NT) and touching (T) devices with a similar / $/$. The colored green area is the channel region damaged by the $\mathrm{He}^{+}$ion beam, while the red area is the non-irradiated $\mathrm{MoS}_{2}$ channel. Scale bars are both $5 \mu \mathrm{m}$. (b) Output and (c) transfer curves of devices with $I_{R}=18 \%$ from the NT and T categories, highlighting the deleterious effect of electrode irradiation on the current injection. (d) Simulation of the $\mathrm{S}$ vacancy yield generated as the $\mathrm{He}^{+}$ion beam penetrates the device at the contact stack, indicating damage to the metal-semiconductor interface. 
may be empirically confirmed with a combination of low-temperature electrical characterization and capacitance measurements $[44,45]$ in future work.

\section{Conclusion}

In summary, we have studied the effects of varying the irradiated channel area of helium ion-treated monolayer $\mathrm{MoS}_{2}$ FETs Introducing a small number of defects into the material (approx. $10 \%$ of irradiated-to-pristine channel area) can serve to improve the charge carrier mobility and the electrical conductance. We found that irradiating the electrode- $\mathrm{MoS}_{2}$ interface was deleterious to the performance of the FET, with a conductance drop noticed for each of the areal irradiation regimes. Our work demonstrates that by tuning the helium ion irradiation strategy, and localizing the exposure to specific sites, the electronic characteristics of on-dielectric $\mathrm{MoS}_{2}$ FETs can be well-controlled in the monolayer limit. Post-metallization irradiations need to be finely controlled to ensure that the hybridized metal-semiconductor interface is not disturbed, otherwise the drive current in ion beam-treated 2D FETs will be limited for certain applications.

\section{Acknowledgements}

We acknowledge D. Keane for helpful discussions and D. Daly for assistance with the helium ion microscope.

\section{Funding}

The following sources of funding are acknowledged: the Leverhulme Trust International Networks Grant (PicoFIB), Science Foundation Ireland (12/TIDA/I2433) and the Irish Research Council (GOIPG/2014/972).

\section{ORCID ${ }^{\circledR}$ iDs}

Jakub Jadwiszczak - https://orcid.org/0000-0002-9518-9402

Pierce Maguire - https://orcid.org/0000-0002-5117-4233

\section{References}

1. Jariwala, D.; Sangwan, V. K.; Late, D. J.; Johns, J. E.; Dravid, V. P.; Marks, T. J.; Lauhon, L. J.; Hersam, M. C. Appl. Phys. Lett. 2013, 102, 173107. doi: $10.1063 / 1.4803920$

2. Baugher, B. W. H.; Churchill, H. O. H.; Yang, Y.; Jarillo-Herrero, P. Nano Lett. 2013, 13, 4212-4216. doi:10.1021/nl401916s

3. O'Brien, M.; McEvoy, N.; Motta, C.; Zheng, J.-Y.; Berner, N. C.; Kotakoski, J.; Elibol, K.; Pennycook, T. J.; Meyer, J. C.; Yim, C.; Abid, M.; Hallam, T.; Donegan, J. F.; Sanvito, S.; Duesberg, G. S. 2D Mater. 2016, 3, 021004. doi:10.1088/2053-1583/3/2/021004

4. Bergeron, H.; Sangwan, V. K.; McMorrow, J. J.; Campbell, G. P.; Balla, I.; Liu, X.; Bedzyk, M. J.; Marks, T. J.; Hersam, M. C. Appl. Phys. Lett. 2017, 110, 053101. doi:10.1063/1.4975064

5. Smithe, K. K. H.; Suryavanshi, S. V.; Muñoz Rojo, M.; Tedjarati, A. D.; Pop, E. ACS Nano 2017, 11, 8456-8463. doi:10.1021/acsnano.7b04100
6. Iberi, V.; levlev, A. V.; Vlassiouk, I.; Jesse, S.; Kalinin, S. V.; Joy, D. C.; Rondinone, A. J.; Belianinov, A.; Ovchinnikova, O. S. Nanotechnology 2016, 27, 125302. doi:10.1088/0957-4484/27/12/125302

7. Klein, J.; Kuc, A.; Nolinder, A.; Altzschner, M.; Wierzbowski, J.; Sigger, F.; Kreupl, F.; Finley, J. J.; Wurstbauer, U.; Holleitner, A. W.; Kaniber, M. 2D Mater. 2017, 5, 011007. doi:10.1088/2053-1583/aa9642

8. Stanford, M. G.; Lewis, B. B.; Mahady, K.; Fowlkes, J. D.; Rack, P. D. J. Vac. Sci. Technol., B: Nanotechnol. Microelectron.: Mater., Process., Meas., Phenom. 2017, 35, 030802. doi:10.1116/1.4981016

9. Fox, D. S.; Zhou, Y.; Maguire, P.; O’Neill, A.; Ó’Coileáin, C.; Gatensby, R.; Glushenkov, A. M.; Tao, T.; Duesberg, G. S.; Shvets, I. V.; Abid, M.; Abid, M.; Wu, H.-C.; Chen, Y.; Coleman, J. N.; Donegan, J. F.; Zhang, H. Nano Lett. 2015, 15, 5307-5313. doi:10.1021/acs.nanolett.5b01673

10. Stanford, M. G.; Pudasaini, P. R.; Gallmeier, E. T.; Cross, N.; Liang, L.; Oyedele, A.; Duscher, G.; Mahjouri-Samani, M.; Wang, K.; Xiao, K.; Geohegan, D. B.; Belianinov, A.; Sumpter, B. G.; Rack, P. D. Adv. Funct. Mater. 2017, 27, 1702829. doi:10.1002/adfm.201702829

11. Jadwiszczak, J.; Keane, D.; Maguire, P.; Cullen, C. P.; Zhou, Y.; Song, H.; Downing, C.; Fox, D.; McEvoy, N.; Zhu, R.; Xu, J.; Duesberg, G. S.; Liao, Z.-M.; Boland, J. J.; Zhang, H. ACS Nano 2019, 13, 14262-14273. doi:10.1021/acsnano.9b07421

12. Liu, Y.; Gao, Z.; Tan, Y.; Chen, F. ACS Nano 2018, 12, 10529-10536. doi:10.1021/acsnano.8b06503

13. Ma, Q.; Odenthal, P. M.; Mann, J.; Le, D.; Wang, C. S.; Zhu, Y.; Chen, T.; Sun, D.; Yamaguchi, K.; Tran, T.; Wurch, M.; McKinley, J. L.; Wyrick, J.; Magnone, K.; Heinz, T. F.; Rahman, T. S.; Kawakami, R.; Bartels, L. J. Phys.: Condens. Matter 2013, 25, 252201. doi:10.1088/0953-8984/25/25/252201

14. Li, Z.; Chen, F. Appl. Phys. Rev. 2017, 4, 011103. doi:10.1063/1.4977087

15. Maguire, P.; Fox, D. S.; Zhou, Y.; Wang, Q.; O'Brien, M.; Jadwiszczak, J.; Cullen, C. P.; McManus, J.; Bateman, S.; McEvoy, N.; Duesberg, G. S.; Zhang, H. Phys. Rev. B 2018, 98, 134109. doi:10.1103/physrevb.98.134109

16. Maguire, P.; Downing, C.; Jadwiszczak, J.; O’Brien, M.; Keane, D.; McManus, J. B.; Duesberg, G. S.; Nicolosi, V.; McEvoy, N.; Zhang, H. J. Appl. Phys. 2019, 125, 064305. doi:10.1063/1.5086366

17. Stanford, M. G.; Pudasaini, P. R.; Cross, N.; Mahady, K.; Hoffman, A. N.; Mandrus, D. G.; Duscher, G.; Chisholm, M. F.; Rack, P. D. Small Methods 2017, 1, 1600060. doi:10.1002/smtd.201600060

18. Stanford, M. G.; Pudasaini, P. R.; Belianinov, A.; Cross, N.; Noh, J. H.; Koehler, M. R.; Mandrus, D. G.; Duscher, G.; Rondinone, A. J.; Ivanov, I. N.; Ward, T. Z.; Rack, P. D. Sci. Rep. 2016, 6, 27276. doi:10.1038/srep27276

19. Yu, Z. G.; Zhang, Y.-W.; Yakobson, B. I. Nano Lett. 2015, 15, 6855-6861. doi:10.1021/acs.nanolett.5b02769

20. Jadwiszczak, J.; O'Callaghan, C.; Zhou, Y.; Fox, D. S.; Weitz, E.; Keane, D.; Cullen, C. P.; O’Reilly, I.; Downing, C.; Shmeliov, A.; Maguire, P.; Gough, J. J.; McGuinness, C.; Ferreira, M. S.; Bradley, A. L.; Boland, J. J.; Duesberg, G. S.; Nicolosi, V.; Zhang, H. Sci. Adv. 2018, 4, eaao5031. doi:10.1126/sciadv.aao5031

21. Bertolazzi, S.; Bonacchi, S.; Nan, G.; Pershin, A.; Beljonne, D.; Samorì, P. Adv. Mater. (Weinheim, Ger.) 2017, 29, 1606760. doi:10.1002/adma.201606760

22. Sangwan, V. K.; Hersam, M. C. Annu. Rev. Phys. Chem. 2018, 69, 299-325. doi:10.1146/annurev-physchem-050317-021353 
23. Shang, M.-H.; Hou, H.; Zheng, J.; Yang, Z.; Zhang, J.; Wei, S.; Duan, X.; Yang, W. J. Phys. Chem. Lett. 2018, 9, 6032-6037. doi:10.1021/acs.jpclett.8b02591

24. Komsa, H.-P.; Krasheninnikov, A. V. Phys. Rev. B 2015, 91, 125304. doi:10.1103/physrevb.91.125304

25. Buchheim, J.; Wyss, R. M.; Shorubalko, I.; Park, H. G. Nanoscale 2016, 8, 8345-8354. doi:10.1039/c6nr00154h

26. Yoon, K.; Rahnamoun, A.; Swett, J. L.; Iberi, V.; Cullen, D. A.; Vlassiouk, I. V.; Belianinov, A.; Jesse, S.; Sang, X.; Ovchinnikova, O. S.; Rondinone, A. J.; Unocic, R. R.; van Duin, A. C. T. ACS Nano 2016, 10, 8376-8384. doi:10.1021/acsnano.6b03036

27. Stanford, M. G.; Noh, J. H.; Mahady, K.; levlev, A. V.; Maksymovych, P.; Ovchinnikova, O. S.; Rack, P. D. ACS Appl. Mater. Interfaces 2017, 9, 35125-35132. doi:10.1021/acsami.7b10449

28. Lin, Z.; McCreary, A.; Briggs, N.; Subramanian, S.; Zhang, K.; Sun, Y.; Li, X.; Borys, N. J.; Yuan, H.; Fullerton-Shirey, S. K.; Chernikov, A.; Zhao, H.; McDonnell, S.; Lindenberg, A. M.; Xiao, K.; LeRoy, B. J.; Drndić, M.; Hwang, J. C. M.; Park, J.; Chhowalla, M.; Schaak, R. E.; Javey, A.; Hersam, M. C.; Robinson, J.; Terrones, M. 2D Mater. 2016, 3, 042001. doi:10.1088/2053-1583/3/4/042001

29. Kaasbjerg, K.; Martiny, J. H. J.; Low, T.; Jauho, A.-P. Phys. Rev. B 2017, 96, 241411. doi:10.1103/physrevb.96.241411

30. Li, S.-L.; Tsukagoshi, K.; Orgiu, E.; Samorì, P. Chem. Soc. Rev. 2016, 45, 118-151. doi:10.1039/c5cs00517e

31. Seo, S.-Y.; Park, J.; Park, J.; Song, K.; Cha, S.; Sim, S.; Choi, S.-Y.; Yeom, H. W.; Choi, H.; Jo, M.-H. Nat. Electron. 2018, 1, 512-517. doi:10.1038/s41928-018-0129-6

32. Cheng, Z.; Cardenas, J. A.; McGuire, F.; Najmaei, S.; Franklin, A. D. IEEE Electron Device Lett. 2016, 37, 1234-1237. doi:10.1109/led.2016.2591552

33. O'Brien, M.; McEvoy, N.; Hallam, T.; Kim, H.-Y.; Berner, N. C.; Hanlon, D.; Lee, K.; Coleman, J. N.; Duesberg, G. S. Sci. Rep. 2014, 4, 7374. doi:10.1038/srep07374

34. Ghorbani-Asl, M.; Kretschmer, S.; Spearot, D. E.; Krasheninnikov, A. V. 2D Mater. 2017, 4, 025078. doi:10.1088/2053-1583/aa6b17

35. Arnold, A. J.; Shi, T.; Jovanovic, I.; Das, S. ACS Appl. Mater. Interfaces 2019, 11, 8391-8399. doi:10.1021/acsami.8b18659

36. Pudasaini, P. R.; Oyedele, A.; Zhang, C.; Stanford, M. G.; Cross, N.; Wong, A. T.; Hoffman, A. N.; Xiao, K.; Duscher, G.; Mandrus, D. G.; Ward, T. Z.; Rack, P. D. Nano Res. 2018, 11, 722-730. doi:10.1007/s12274-017-1681-5

37. Dhall, R.; Li, Z.; Kosmowska, E.; Cronin, S. B. J. Appl. Phys. 2016, 120, 195702. doi:10.1063/1.4967398

38. Yoo, G.; Hong, S.; Heo, J.; Kim, S. Appl. Phys. Lett. 2017, 110, 053112. doi:10.1063/1.4975626

39. Kretschmer, S.; Maslov, M.; Ghaderzadeh, S.; Ghorbani-Asl, M.; Hlawacek, G.; Krasheninnikov, A. V. ACS Appl. Mater. Interfaces 2018, 10, 30827-30836. doi:10.1021/acsami.8b08471

40. Choi, H. H.; Cho, K.; Frisbie, C. D.; Sirringhaus, H.; Podzorov, V. Nat. Mater. 2018, 17, 2-7. doi:10.1038/nmat5035

41. Ziegler, J. F.; Ziegler, M. D.; Biersack, J. P. Nucl. Instrum. Methods Phys. Res., Sect. B 2010, 268, 1818-1823. doi:10.1016/j.nimb.2010.02.091

42. Kim, C.; Moon, I.; Lee, D.; Choi, M. S.; Ahmed, F.; Nam, S.; Cho, Y.; Shin, H.-J.; Park, S.; Yoo, W. J. ACS Nano 2017, 11, 1588-1596. doi:10.1021/acsnano.6b07159
43. Arutchelvan, G.; Lockhart de la Rosa, C. J.; Matagne, P.; Sutar, S.; Radu, I.; Huyghebaert, C.; De Gendt, S.; Heyns, M. Nanoscale 2017, 9, 10869-10879. doi:10.1039/c7nr02487h

44. Moon, B. H.; Han, G. H.; Kim, H.; Choi, H.; Bae, J. J.; Kim, J.; Jin, Y.; Jeong, H. Y.; Joo, M.-K.; Lee, Y. H.; Lim, S. C. ACS Appl. Mater. Interfaces 2017, 9, 11240-11246. doi:10.1021/acsami.6b16692

45. Kim, H.-J.; Kim, D.-H.; Jeong, C.-Y.; Lee, J.-H.; Kwon, H.-I. IEEE Electron Device Lett. 2017, 38, 481-484. doi:10.1109/led.2017.2673854

\section{License and Terms}

This is an Open Access article under the terms of the Creative Commons Attribution License (https://creativecommons.org/licenses/by/4.0). Please note that the reuse, redistribution and reproduction in particular requires that the authors and source are credited.

The license is subject to the Beilstein Journal of Nanotechnology terms and conditions: (https://www.beilstein-journals.org/bjnano)

The definitive version of this article is the electronic one which can be found at: https://doi.org/10.3762/bjnano.11.117 